\title{
Nurturing empathy and compassion: what might the neurosciences have to offer?
}

\author{
Manuel J Costa \& Patrício Costa
}

The assumption that the valuing of compassion and empathy by physicians is important in patient care has been part of medical education for many years. Recent empirical evidence suggests that physician empathy is therapeutic in itself. ${ }^{1,2}$ However, medical education is still searching for fitfor-purpose curricula that adequately nurture empathy and compassion in students. Such curricula should ensure that medical students graduate with adequate knowledge and, above all, practise medicine according to those values. There are multiple challenges facing the development of such curricula. Empathy and compassion are multidimensional constructs, have complex and sometimes contradictory definitions, ${ }^{3}$ and are very sensitive to contextual influences in practice (including those of the deleterious 'hidden curriculum, ${ }^{4}$ ). Both constructs are also conditioned by physician well-being, ${ }^{5}$ which makes the fostering of well-being and learning to self-care important objectives of an empathy and compassion curriculum.

Medical education is still searching for fit-for-purpose curricula that adequately nurture empathy and compassion in students

Braga, Portugal

Correspondence: Manuel João Costa, Life and Health Sciences Research Institute (ICVS), School of Health Sciences, University of Minho, Gualtar Campus, Braga 4710-057, Portugal. Tel: 00351253 604805;

E-mail: mmcosta@ecsaude.uminho.pt

doi: $10.1111 /$ medu.12980
Learning what other disciplines have to say about the topics is therefore timely. Over the past 15 years, the dominant research paradigm used to study medical students' empathy and compassion has been positivism. Most research has focused on the development and use of instruments and on the identification of statistical correlations or of variations in scores over time. ${ }^{6,7}$ Alternative paradigms that consider socio-cultural contexts are necessary to offer education developers a theory-based framework and to generate concrete recommendations for the design of a fit-for-purpose empathy and compassion curriculum. In this issue of Medical Education, Dr Beth Lown sheds new light on this debate by offering a social neurosciences perspective. $^{8}$

Alternative paradigms that consider socio-cultural contexts are necessary to offer education developers a theory-based framework and to generate concrete recommendations

The neurosciences offer a refreshing biological stance for education research in this area. Knowledge from this discipline is relevant to the realm of empathy and compassion in that it refers to the modulation of brain networks involved in the processing of affective and motivational experiences. For example, there is a specific brain core neural network that is responsive to emotions designated as belonging to the 'pain-empathy network'. This network is activated in response to one's own personal experiences or to observations of others, through voluntary or involuntary processes that neuroscientists have named 'affective empathy' and 'cognitive empathy'. There are modulators in clinical contexts that influence both processes through partially overlapping pathways. Interestingly, the existence of two partially overlapping brain processes resonates with definitions of empathy in medical education that explicitly refer to a 'cognitive' and an 'affective' dimension. The indication that brain modulation imaging studies may inform educational interventions is intriguing as a path towards novel research approaches. For example, if neurofeedback training can contribute to the regulation of emotional processes in mood disorders, ${ }^{9,10}$ perhaps it can also be applied to students or doctors.

The existence of two partially overlapping brain processes resonates with definitions of empathy in medical education that explicitly refer to a 'cognitive' and an 'affective' dimension

Interestingly, the brain modulators described in Dr Lown's article, ${ }^{8}$ which act through the deactivation of the neural networks involved in understanding and responding to another person's experiences, resemble important elements of the hidden curriculum. These include circumstances that divert a student's attention away from the patient, the devaluation of the patient, or repeated exposure to others in pain. Furthermore, the hardwiring of the brain is sensitive to elements implicated in the per- 
sonal distress of doctors and students, such as a relative inability to understand or to regulate one's own emotions or to respect the self-other boundaries. This further suggests that bridging the biological and educational worlds can help to inform the design of educational interventions. In fact, through the integration of concepts from psychology - cognitive and social and research on the social neuroscience of empathy and compassion, Table 1 in Dr Lown's article ${ }^{8}$ offers a menu of curriculum goals, interventions and resources that medical educators can put to the test. Some interventions relate to the valuing of, attention to and sensitisation for empathy and compassion. These can be built into curricula and integrated with the corresponding learning objectives, activities and assessment programmes. As there is no reason why early courses should not teach and assess these components explicitly, the learning of these topics can start in the early years. The implementation of the other interventions listed, including those that aim to educate students to understand and develop their abilities to self-care, to deal with blurred selfother borders, and to regulate selfemotions in response to others' emotions will probably be more challenging. Effective emotional education would require opportunities, self-reflection and feedback focused on the emotional process itself. It would also require adequate debriefing encounters with simulated and real patients.

The brain modulators described in $\mathrm{Dr}$ Lown's article, which act through deactivation of the neural networks involved in understanding and responding to other's experiences, resemble important elements of the "hidden curriculum"
The most interesting developments for medical education highlighted by Dr Lown's provision of access to the neurosciences $^{8}$ are perhaps the broader implicit messages conveyed by this article. The realisation that understanding compassion and empathy in medical education should be informed by the social neurosciences might offer a starting point for transdisciplinary collaborative research efforts. Generalisations of the evidence from laboratory studies to authentic settings must always be cautious as the effects of contextual and cultural circumstances are paramount. Research is necessary to evaluate the practical significance of interventions and also to clarify the influences of contextual and individual specificities. Under all circumstances, the neurosciences provide new opportunities to accelerate the development of fit-for-purpose curricula to nurture the empathy and compassion of students and of practising doctors. Hardwired with professionalism and with the other values of the profession, such curricula will be important in guaranteeing both the competence and the caring dimensions of medical graduates.

The realisation that understanding compassion and empathy in medical education should be informed by social neurosciences might offer a starting point for trans-disciplinary collaborative research effort

\section{REFERENCES}

1 Hojat M, Louis D, Markham F, Wender R, Rabinowitz C, Gonnella J. Physicians' empathy and clinical outcomes for diabetic patients. Acad Med 2011;86 (3):359-64.

2 Rakel D, Barrett B, Zhang Z, Hoeft T, Chewning B, Marchand L, Scheder J. Perception of empathy in the therapeutic encounter: effects on the common cold. Patient Educ Couns 2011;85 (3):390-7.

3 Mercer SW, Reynolds WJ. Empathy and quality of care. BrJ Gen Pract 2002;52 (Suppl):9-13.

4 Hafferty F. Beyond curriculum reform. Acad Med 1998;73 (4):403-7.

5 West C, Huschka M, Novotny P, Sloan J, Kolars J, Habermann T, Shanafelt TD. Association of perceived medical errors with resident distress and empathy: a prospective longitudinal study. JAMA 2006;296 (9):1071-8.

6 Hemmerdinger J, Stoddart S, Lilford R. A systematic review of tests of empathy in medicine. $B M C$ Med Educ 2007;7 (1):24.

7 Neumann M, Edelhäuser F, Tauschel D, Fischer M, Wirtz M, Woopen C, Haramati A, Scheffer C. Empathy decline and its reasons: a systematic review of studies with medical students and residents. Acad Med 2011;86 (8):996-1009.

8 Lown BA. A social neuroscienceinformed model for teaching and practising compassion in health care. Med Educ 2016;50 (3):332-42.

9 Hammond DC. Neurofeedback treatment of depression and anxiety. J Adult Dev 2005;12 (2-3):131-7.

10 Stoeckel LE, Garrison KA, Ghosh $\mathrm{S}$ et al. Optimising real time fMRI neurofeedback for therapeutic discovery and development. Neuroimage Clin 2014;5 (1):245-55. 